\title{
Contacto precoz piel a piel: efecto sobre los parámetros fisiológicos en las cuatro horas posteriores al parto en recién nacidos de término sanos
}

\author{
Hernán Víllalón $\mathrm{U}^{1}{ }^{\text {; }}$ Patricia Alvarez $\mathrm{C}^{1}$; Eliana Barría $\mathrm{H}^{2}{ }^{2}$; Daisy Caneleo $\mathrm{H}^{2}$ \\ Luisa Cartillo $\mathrm{M}^{2}$; Silvia Durán $\mathrm{G}^{2}{ }^{2}$; Carmen Luz Feres $\mathrm{R}^{2}$; Patricia Mansilla A. $^{2}$; \\ Azucena Nayarro $\mathrm{C}^{2}{ }^{2}$; Cristjna Olivares $\mathrm{G}^{2}$; Victoria Torres $\mathrm{R} .^{2}$ \\ Effect of early skin-to-skin contact on temperature \\ regulation, heart rate and respiratory rate in healthy, full-term newborns
}

\begin{abstract}
Eariy skin-to-skin contact as a means of warmth and its effect on heart and respiratory rate during four hours following birth, was analized. Ninety two healthy full-term newborns were studied through a randomized prospective controlled trial, along december 1991 throughout febuluary $\uparrow 992$ at the regional hospital of Covhaique, Chile (Western Patagoniak. Forty seven infants were placed prone, skin-to-skin between their mother's breasts during four hours, and both were left in the maternity ward. Three mothers rejected the method because of significant symptoms associated with labor Control group underwent classical management in nurserv. Environmental tem. perate ranged between 19 to $25^{\circ} \mathrm{C}\left(21,66 \pm 1.62^{\circ} \mathrm{C}\right.$ in the maternity wards while in nursery, for control infants, it was $26 \cdot 310\left(28.21 \pm 1.28^{\circ} \mathrm{C}\right) p<0.01$. No differences in the ability to regulate bodv temperature were found in both groups of infants. Heart and respiratory rates were similar during the four hours study period. Forty-four mothers in the skin-to-skin contact group $\{93.6 \%\}$, and $100 \%$ of participating personnel, expressed a very good opinion about the experience. In conclusion, the method can be used safely in healthy, full-term newborn with environmental temperature as low as $19^{\circ} \mathrm{C}$. It might be considered as a very interesting and encouraging experience.
\end{abstract}

(Key words: Newborn, skin-to-sk in contact, temperature regulation, heart rate, respiratorv rate.)

En el Hospital Regional de Coyhaique, el periodo de adaptación inmediata del recién nacido se realiza en una sala de atención inmediata (SAI), donde se le proporciona un ambiente térmico adecuado y se controla su evolución durante cuatro horas. Posteriormente el niffo es llevado a su madre, en caso de mostrar estabilidad térmica y no presentar alteraciones durante su periodo adaptativo.

Se ha sugerido que el contacto precoz entre el recién nacido y su madre tiene influencia positiva en la relación entre ambos, principalmente en los cuatro días siguientes al parto. Además, tiene un atractivo natural y ayuda a

1. Unidad de Cuidados Intensivos Pediátricos y Neonatales, Hospital Regional Coyhaique, XI Región.

2. Matrona. Servicio de Maternidad. Hospital Regional Coyhajque, XJ Región. superar los sentimientos de frustración que produce la separación del recién nacido y sus padres, principalmente en prematuros ${ }^{1,2}$.

El contacto piel a piel reduce la actividad motora, aumenta los periodos de sueño y disminuye el gasto térmico del recién nacido ${ }^{3}$. Considerando estos buenos resultados, se evaluó la factibilidad de aplicar la experiencia en nuestro hospital a través de un estudio prospectivo controlado, ya que las diferentes condiciones climáticas que caracterizan su medio pudieran impedir el empleo del método en la localidad. E] objetjo de este estudio fue describir la evolución têrmica y cardiorrespiratoria durante las primeras cuatro horas posteriores al parto, du. rante el contacto precoz prolongado, de piel a piel (CPPP), entre el recién nacido y su madre y, además, evaluar la aceptación del método por parte de las madres y el personal de salud participante. 


\section{Pacientes y Métodos}

Pacientes. Se estudiajon 92 recién nacidos y sus madres, en el servicio de Maternidad del Hospital Regional de Coyhaique, en el periodo comprendido por los meses de diciembre de 1991 y febrero de 1992. Los recjén nacidos incluidos tenían una edad gestacional pediátrica de 38 a 41 semanas; peso de nacjmiento entre 3000 y $4000 \mathrm{~g}$; Apgar al primer y quinto minutos gual o superior a 7 y eran producto de embarazo normal y parto eutócico vaginal. Se exchuyeron los recjén nacidos con antecedentes de rotura de membrana superior a 12 horas, los que - por sospecha de infección bacteriana perinatal- tenian alteraciones sugerentes de elta en el hemograma (según criterios de Radwell) o cultivo positivo o ambos, los recién nacidos con malformaciones, dificultad respiratoria, cardiopatías, hipoglicemia. Se excluyeron también dos recién nacjdos del grupo control con taquipnea mayor de 80 - min que no se recuperó después de dos horas de ob. servación.

Métodos. La muestra se distribuyó en forma aleatoria resultando 47 recién nacidos destinados al grupo de CPPP y 45 para control. Se registró información de edad, ruralidad, paridad y escolaridad de las madres. La paridad se subclasificó en primigestas, mult íparas de 1 a 2, multíparas de 3 o más. La escolaridad se calificó en inferior a $6^{\circ}$ año básico, entre $7^{\circ}$ año básico y $4^{\circ}$ medio y educación universitaria o técnico-profesional.
También se registraron datos del embarazo y parto (drogas, infusión ocitócica, anestesia, desaceleración de latidos cardiofetales, Apgar al primer y quinto minutos, tiempo de rotura de membranas), características del recién nacido (sexo, peso de nacimiento, edad gestacional pediátrica estimada según Parkin) y las variables en estudio: temperatura ambiente, medida en la SAl y salas de la maternidad -mediante un temómetrc ambiental marca Werbe-; temperatura axilar -mediante termómetro clínico durante 5 minutos-al nacimiento y cada hora en las siguiente 4 horas; frecuencia cardíaca (FC) y respiratoria (FR), contadas durante un minuto, al nacimiento $y$ cada hora durante las siguientes 4 horas. Todo lo anterior se describe en la tabla 1.

En el momento del alta se preguntó directamente a las madres que experimentaron el CPPP su opinión al respecto, clasificándola como buena, regular o rechazo al método. También se pioió la opinión del personal de salud participante, cuyas respuestas fueron clasificadas de la misma manera. Una anplia gama de personal participó en los registros (matronas, auxiliares de enfermería), por to que se puso especial énfasis en la supervisión para evitar diferencias en el trato a ambos grupos.

En el grupo control, después del nacimiento, secado y mediciones antropométricas, los recién nacidos fueron restidos $y$ dejados en la SAI durante un período de cuatro horas donde se realizaron las evaluaciones que se

Tabla 1

Caracter ísticas generales de 47 recién nacidos sometidos a contacto precoz piel a piel con su madre y 45 recién nacidos controles

\begin{tabular}{|c|c|c|c|c|c|c|c|}
\hline Sexo & & \multicolumn{2}{|r|}{$\begin{array}{l}\text { Grupo CPPP } \\
\text { (n: } 47)\end{array}$} & \multicolumn{3}{|c|}{$\begin{array}{l}\text { Grupo control } \\
\text { (n: 45) }\end{array}$} & p \\
\hline Masculino & $25 / 47$ & & $(53,2 \%)$ & $20 / 45$ & & $(44,4 \%)$ & ns \\
\hline Femenino & $22 / 47$ & & $(46,8 \%)$ & $25 / 45$ & & $(55,6 \%)$ & ns \\
\hline Peso de nacimiento (g) & 3428 & \pm & 236 & 3404 & \pm & 277 & ns \\
\hline Edad gestacional (sem) & 39,1 & \pm & 0,8 & 39,4 & \pm & 0,7 & ns \\
\hline \multirow{2}{*}{\multicolumn{8}{|c|}{ Escolaridzd }} \\
\hline & & & & & & & \\
\hline Menor $7^{\circ}$ básico & $17 / 47$ & & $(36,2 \%)$ & $10 / 45$ & & $(22,24)$ & ns \\
\hline $7^{\circ}$ básico $-4^{\circ}$ medio & $26 / 47$ & & $(55,3 \%)$ & $24 / 45$ & & $(53,3 \%)$ & ns \\
\hline Univ./Técn. profesional & $4 / 47$ & & $(8,5 \%)$ & $5 / 45$ & & $(11,1 \%)$ & ins \\
\hline \multicolumn{8}{|l|}{ Paridad } \\
\hline Primigesta & $13 / 47$ & & $(27,7 \%)$ & $15 / 45$ & & $(33,3 \%)$ & ns \\
\hline M1-M2 & $21 / 47$ & & $(44,7 \%)$ & $24 / 45$ & & $(53,3 \%)$ & ins \\
\hline$>\mathrm{M} 3$ & $13 / 47$ & & $(27,7 \%)$ & $6 / 45$ & & $(13,3 \%)$ & ns \\
\hline Ruralidad & $16 / 47$ & & $(34,0 \%)$ & $15 / 45$ & & $(33,3 \%)$ & ns \\
\hline Drogas en embarazo & & -- & & & - & & \\
\hline Infusión ocitócica & $11 / 47$ & & $(23,4 \%)$ & $10 / 45$ & & $(22,2 \%)$ & ns \\
\hline \multicolumn{8}{|l|}{ Anestesia } \\
\hline Peridural & $5 / 47$ & & $(10,6 \%)$ & $4 / 45$ & & $(8,8 \%)$ & ns \\
\hline Anestesia local & $28 / 47$ & & $(59,6 \%)$ & $29 / 45$ & & $(64,4 \%)$ & ns \\
\hline Sin anestesiz & $14 / 47$ & & $(29,0 \%)$ & $12 / 45$ & & $(26,8 \%)$ & ns \\
\hline Desaceleración LCF & $4 / 47$ & & $(8,5 \%)$ & $3 / 45$ & & $(6,7 \%)$ & ns \\
\hline Tipo rotura membrana (h) & 3,03 & \pm & 2,78 & 2,87 & \pm & 3,45 & ns \\
\hline
\end{tabular}

CPPP: Contacto precoz piel a piel. LCF: Latidos cardíacos fetales. 
indican en las tablas 1 y 2 . En el grupo de contacto procoz prolongado pjel a pjel (CPPP), posterior al nacjmiento, secado y mediciones antropométricas, los recién nacidos, vestidos sólo con un pañal, fueron entregados a sus madres, puestos entre las mamas y cubiertos sólo por una sábana. De esta manera se inicia precozmente un contacto piel a piel entre las superficies ventrales de la madre y su hijo y la succión del pezón. Terminada la episiorrafia, el contacto se prolongó por cuatro horas en las salas de la maternidad, donde se realizaron las evaluaciones aludidas.

En el análisis estad istico de los resultados se emplearon las pruebas de chi cuadrado para comparacion de proporciones y porcentajes, análisis de varianza de prueba t de Student para promedios y se estableció el límite de confianza en menos de $5 \%$.

\section{Resultados}

En la tabla 1 se resumen las caracteristicas de los grupos CPPP y control. Ambos fueron com. parables en distribución por sexo, peso de nacimiento y edad gestacional de los recién nacidos. Las madres también fueron comparables en edad, paridad, ruralidad, infusión de ocitócicos, empleo y tipo de anestesia, desaceleración de latidos cardíacos fetales en el período expulsivo y tiempo de rotura de membranas. En la escolaridad se observaron algunas diferencias que no alcanzaron significación estadistica, ya que en el grupo estudiado se observó 36,2\% de baja escolaridad (sex to básico o menos), incluso hubo dos casos de analfabetismo $(5,0 \%)$, mientras que en el grupo control sólo $22,2 \%$ de las madres presentó baja escolaridad y ninguna analfabetismo; pero estas diferencias no tienen una magnitud que influya en el resultado de las variables analizadas.

En la tabla 2 se resumen los resultados obtenidos con las variables estudiadas. La temperatura ambiental fue significativamente más baja para el grupo CPPP que para los controles, con márgenes de $19^{\circ} \mathrm{C}$ a $25^{\circ} \mathrm{C}$ y de $26^{\circ} \mathrm{C}$ a $31^{\circ} \mathrm{C}$, respectivamente, mientras la temperatura corporal mostró sólo leves diferencias, principalmente 1 h después del nacimiento, siempre dentro del rango normal y sin significación estadística. Sólo en dos recién nacidos de cada grupo se registraron temperaturas corporales inferiores a $36,5^{\circ} \mathrm{C}$, que en el grupo CPPP coincidieron con temperaturas ambientales de $19^{\circ} \mathrm{C}$. El comportamiento de las frecuencias cardíacas y respiratorias fue similar en ambos grupos de recién nacidos. Tampoco se encontraron diferencias al analizar la capacidad de temorregulación del grupo CPPP en bandas de temperatura ambien.

Tabla 2

Evolución de la temperatura, frecuencia cardiaca, frecuencia tespiratoria en 44 RN manejados con contacto precoz pie] a piel y 45 controles

\begin{tabular}{|c|c|c|c|c|c|c|c|}
\hline \multirow[b]{2}{*}{$\begin{array}{l}\text { T. ambiental }\left({ }^{\circ} \mathrm{C}\right) \\
\text { T. axilar }\left({ }^{\circ} \mathrm{C}\right)\end{array}$} & \multirow[b]{2}{*}{21,66} & \multirow{2}{*}{\multicolumn{2}{|c|}{$\begin{array}{c}\text { Grupo CPPP } \\
(\mathrm{n}: 44) \\
1,63\end{array}$}} & \multicolumn{3}{|c|}{$\begin{array}{c}\text { Grupo control } \\
\text { (n: } 45)\end{array}$} & \multirow{2}{*}{$\begin{array}{c}\mathbf{p} \\
<0,01\end{array}$} \\
\hline & & & & 28,21 & \pm & 1,28 & \\
\hline Nacimiento & 37,09 & \pm & 0,27 & 37,01 & \pm & 0,31 & ns \\
\hline Primera hora & 36,84 & \pm & 0,32 & 37,06 & \pm & 0,39 & ns \\
\hline Segunda hora & 37,00 & \pm & 0,28 & 37,10 & \pm & 0,39 & ns \\
\hline Tercera hora & 36,97 & \pm & 0,20 & 37,10 & \pm & 0,18 & ns \\
\hline Cuarta hora & 36,86 & \pm & 0,26 & 36,98 & \pm & 0,19 & ns \\
\hline \multicolumn{8}{|c|}{ F. card iaca (lat./min.) } \\
\hline Nacimiento & 152,1 & \pm & 8,2 & 145,1 & \pm & 8,3 & ns \\
\hline Primera hora & 144,3 & \pm & 9,6 & 147,4 & \pm & 9,9 & ns \\
\hline Segunda hora & 144,4 & \pm & 7,3 & 143,2 & \pm & 8,0 & ns \\
\hline Tercera hora & 146,0 & \pm & 9,9 & 146.2 & \pm & 9,6 & ns \\
\hline Cuarta hora & 144,0 & \pm & 8,1 & 140,9 & \pm & 10,8 & ns \\
\hline \multicolumn{8}{|c|}{ F. respiratoria (res/min) } \\
\hline Naciniento & 49,2 & \pm & 7,3 & 50,7 & \pm & 6,4 & sts \\
\hline Primera hora & 48,9 & \pm & 8,0 & 47,9 & \pm & 6.4 & $\mathrm{~ns}$ \\
\hline Segunda hora & 47,7 & \pm & 8,9 & 46,0 & \pm & 6,3 & ns \\
\hline Tercera hora & 47,0 & \pm & 6,4 & 48,0 & $t$ & 7,2 & ns \\
\hline Cuarta hora & 46,4 & \pm & 6,9 & 44,9 & $t$ & 7,3 & $\pi \mathrm{s}$ \\
\hline Quejido espiratorio & $3 / 44$ & & $(6,8 \%)$ & $2 / 45$ & & $(4,4 \%)$ & $\mathrm{ns}$ \\
\hline
\end{tabular}

CPPP: Contacto precoz piel a piel. T: Temperatura. F: Frecuencia. 
tal de 19 a 20,21 a 22 y 23 o más $C^{\circ}$. En $6,8 \%$ de los recién nacidos manejados con contacto precoz piel a piel y $4,4 \%$ de los controles se registró quejido espiratorio transitorio y de corta duración, diferencia no significativa.

El método fue aceptado por 44 de las $47 \mathrm{ma}$ dres estudiadas $(93,6 \%)$, calificandolo de bueno o excelente. Los tres rechazos se atribuyeron a vómitos abundantes postparto (un caso), lipotimia materna (un caso), agotamiento (un caso). Todo el personal de salud participante calificó como excelente el procedimiento estudiado.

\section{Comentario}

Los resultados publicados anteriormente mostraban que el CPPP es un buen método para conservar la temperatura en recién nacido de bajo peso de nacimiento en unidades neonatológicas (con ambiente térmico neutral) ${ }^{4-6}$ o ambula. torios y con temperatura ambiental de tipo tropical $\left(22 \text { a } 32^{\circ} \mathrm{C}\right)^{7}$. Los resultados obtenidos en este estudio permiten aplicar el metodo con seguridad en recién nacidos de término, sanos, productos de embarazo y parto normales, cuando la temperatura ambiental es igual o superior a $19^{\circ} \mathrm{C}$. Bajo ésta debe evaluarse el método con abrigo adicional para evitar pérdidas calóricas excesivas (gorro y cobertura con frazadas).

La estabilidad de la evolución de las frecuen. cias cardíaca y respiratoria es concordante con ta de la temperatura corporal y hay estudios que apoyan el uso de la FC como medida de gasto energético ${ }^{3}$. Por otra parte, no se han observado diferencias en los resultados obtenidos con monitoría continuada respiratoria y de la satura. ción arterial de oxígeno entre recién nacidos ma. nejados con contacto piel a piel y los mantenidos en incubadora ${ }^{8}$.

El método tuvo una excelente acogida entre las madres, ya que incluso en los casos de complicaciones en el puerperio inmediato la mayoría de ellas se negó a separarse de su hijo, excepto en casos de molestias muy acentuadas. Se destaca también la aceptación del método entre el personal de salud, ya que a pesar del número de participantes no hubo opiniones negativas ni de disconformidad en relación al procedimiento. El contacto precoz (en sala de partos) y prolongado durante 4 horas piel a piel entre madre y recièn nacido es seguro y aconsejable, ya que su beneficio en el desarrollo de la relación madre e hijo ha sido demostrado en diversos estudios y no debe ser interferido por normas hospitalarias". Sus efectos sobre parámetros, como la lactancia materna, seguridad y asociación con infecciones, deben ser analizados también, ya que los resultados de este trabajo apoyan su aplicación en forma ampliada, lo que permite la realización de estudios tendientes a observar otros beneficios, efectos y complicaciones del método en nuestros pacientes. Por último, nos parece aconsejable fomentar una interacción madre-hijo precoz, natural y gratificante.

\section{Resumen}

Se analiza el contacto precoz piel a piel (CPPP) como medio de conservación de temperatura y su efecto en las frecuencias cardíacas y respira. torias durante las cuatro horas posteriores al nacimiento. Se realizó un estudio prospectivo controlado con selección aleatoria, en el que fueron incluidos 92 recién nacidos de término, sanos, en el periodo diciembre 1991-febrero 1992, en el Hospital Regional de Coyhaique. Cuarenta y siete recién nacidos fueron entregados a sus madres de inmediato para ser ubicados sobre el pecho de ellas, en contacto piet a piel durante cuatro horas. Sólo tres madres rechazaron el método, debido a sufrir molestias significativas. El grupo control recibió el manejo habitual en sala de atención inmediata. El rango de temperatura ambiental fue, para los niños manejados con contacto piel a piel, de 19 a $25^{\circ} \mathrm{C}$ $\left(21,66 \pm 1,63^{\circ} \mathrm{C}\right)$ y 26 a $31^{\circ} \mathrm{C}\left(28,21 \pm 1,28^{\circ} \mathrm{C}\right)$ para los controles ( $p<0,01$ ), a pesar de lo cual no se encontraron diferencias en la capacidad de termorregulación, tampoco en la evolución de frecuencia cardíaca y respiratoria. La mayoría de las madres $(93,6 \%)$ y todo el personal de salud participante expresó una excelente opinión de la experiencia. El método puede ser utilizado con seguridad en recién nacidos de término sanos con temperatura ambiental superior a $19^{\circ} \mathrm{C}$.

(Palabras claves: Recién nacido inmediato, contacto piel a piel, temperatura corporal, frecuencia cardiaca, frecuencia respiratoria.)

\section{Referencias}

1. Widstrom $A M$, Whatberg $V$, Matthiesen $A S$ et al.: Short term effects of early suckling and touch of 
the nipple on maternal behavious. Early Hum Dev $1990 ; 21: 153-163$.

2. Whtt elaw $A$ : Crianza tipo canguro: ¿Solamente una bonita experiencia o un avance importante para los prematuros? Pediatrics (ed. esp.) 1990; 29: 171 . 172.

3. Lidington SM: Energy conservation during skinto-skin contact between premature infants and their mothers. Heart Lung 1990; 19: 445-451.

4. Ludington Hoe SM, Hadeed AJ, Anderson GC: Physiologic responses to skin-to-skin contact it hospitalized premature infants. J Perinatol 1991; 11 : 19-24.

5; Acolet $D$, Sleath $K$, whitelaw A: Oxygenation, heart rate and temperature in very low birth weight infants during skin-to-skin contact with their mothers. Acta Paediatr Sand 1989; 78: 189-193.

6. Anderson GC: Skin-to-skin kangaroo care in Western Europe. Am J Nutsing 1989; 89: 662-666.

7. Mondlane RP, De Graca AM, Ebrahim GJ: Skinto-skin contact as a method of body wammth for infants of low birth weight. I Trop Pediatr 1989; 35: 321-326.

8. Bosques EP. Brady JP, Affonso DD et al,: Continuous physiological measurements of kangaroo versus incubator care in a tertiary level nursety. Pediatr Res 1988; $23: 402$.

9. Radrigón ME, Juez G, Coronel F: Alimentación en el primer año de viđa. Rev Chil Pediatr 1989; 60 (supl. 1): 9-19.

\section{AVISO A LOS AUTORES}

Por acuerdo del Comité Editorial, la Revista Chilena de Pediatria devolverá sin tramitar todos los trabajos que no den estricto cumplimiento al Reglamento de Publicaciones y a las Instrucciones de los autores que se editan en cada número de la Revista. 